\title{
The long and the short of it-the temporal effects of renal lithium exposure are beginning to be unravelled
}

L

ithium has a long history of use in patients with chronic psychological and neurological conditions, such as bipolar disorder and Alzheimer disease. Although lithium treatment has been shown to be neuroprotective, its effects on the kidney have been less positive. Chronic lithium exposure is associated with nephrogenic diabetes insipidus (NDI), which is characterized by polyuria and excessive thirst. Now, researchers in the Netherlands have elucidated that lithium affects principal cell cycling, arresting cells at the G2/M checkpoint to induce renal injury. At the same time, a group led by Rujun Gong at the Brown University School of Medicine, USA, has shown that lithium promotes renal recovery following acute kidney injury (AKI).

"We were interested to know how lithium causes NDI and approached this question from a combined molecular, cell biological and integrative physiological angle," explains Peter Deen, lead investigator of the first study. Together with his collaborator Theun de Groot, their team of researchers treated cultured mouse collecting duct cells with lithium for up to 11 days. Previous work on the effects of lithium on renal principal cells had shown that the drug induced proliferation. Indeed, the researchers observed an abundance of the S/G2 phase marker proliferating cell nuclear antigen (PCNA), consistent with these reports. However, "the first surprising discovery for us was that treating collecting duct cells in vitro led to a significant increase in G2/M-phase-arrested cells," comments Deen, as evidenced by an increase in the levels of phosphorylated histone $\mathrm{H} 3$, a marker of $\mathrm{G} 2 / \mathrm{M}$ phase.

This seemingly contradictory finding could perhaps explain the reduced number of principal cells evident in the collecting ducts of lithium-treated animals compared with controls. "The assumption is that nuclear PCNA staining is synonymous with cell division," continues Deen. "Our data revealed that, although PCNA does

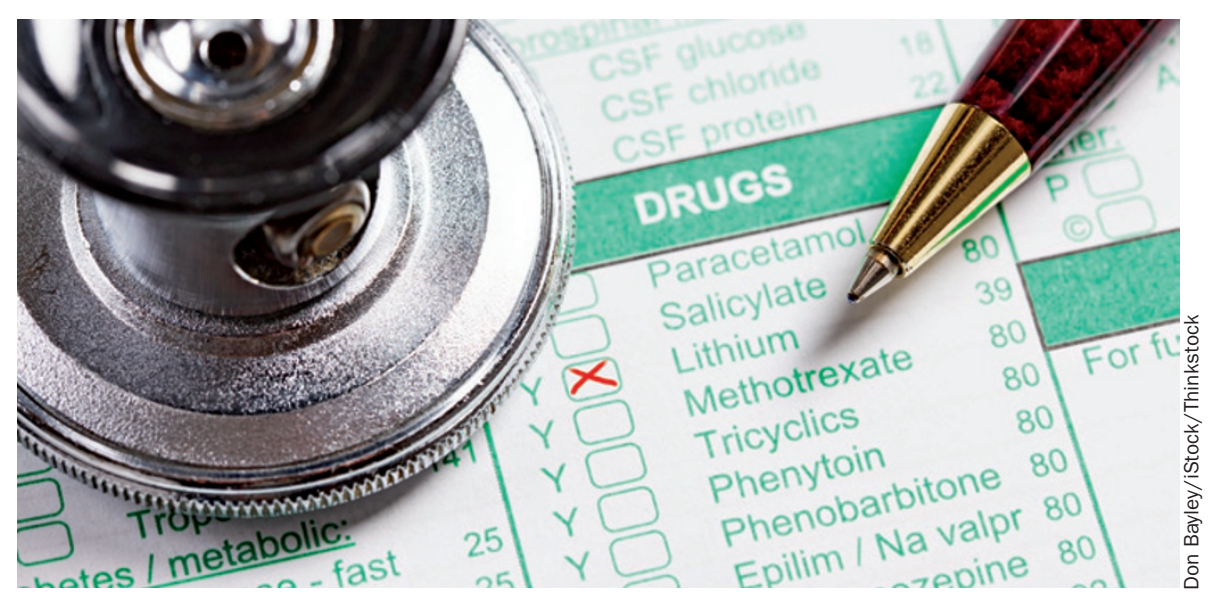

indicate initiation of cell division, it does not necessarily mean division through the whole cell cycle, as it also stains cells that have stopped dividing in the G2/M phase." That is, the cells were getting 'stuck' at the G2/M checkpoint, expressing phosphorylated histone H3 and PCNA.

"After that, we of course hoped that our in vitro data would be supported by the in vivo data, which appeared to be the case."

Clinically, around $60 \%$ of patients with lithium-induced NDI develop cysts, some of whom will go on to develop chronic kidney disease (CKD). "As many renal disorders that start with cyst formation lead to CKD, lithium-induced NDI is a good model to get more insight in CKD development," explains Deen.

While these findings could conceivably support the rational design of therapies to prevent or treat NDI caused by long-term lithium use, those of Gong and colleagues place short-term lithium use firmly in the realm of treatment rather than cause.

"Research has shown that lithium has a general and pro-reparative effect on the kidney," says Gong. "Lithium selectively targets GSK3 $\beta$, a key signalling molecule that plays a part in the pathogenesis of multiple human diseases, including AKI." The doses typically needed for lithium to cross the blood-brain barrier are high, and lead to the toxicity at the focus of
Deen's work. "We demonstrated for the first time that delayed administration of a single low dose of lithium-only one-third to onehalf of the psychiatric dose-following AKI in mice promotes renal tubular epithelial repopulation and kidney repair, and accelerates recovery of kidney function."

In their cisplatin mouse model of AKI, elevated serum creatinine was measured and tubular cell death was evident. At day 3 after the injury, signs of reversal were evident. However, if given lithium on day 3 , the rate of recovery of the mice markedly improved compared with that of untreated controls. Similar improvements to recovery after ischaemic injury were evident in another model.

Mechanistically, lithium blocks GSK3 $\beta$ in the injured kidney, which enhances the expression of pro-proliferative molecules, including cyclin D1, c-Myc and hypoxiainducible factor-1a. "We hope to carry out a pilot clinical trial to define the optimal dose of lithium that promotes recovery of kidney function in patients with AKI," concludes Gong.

Mina Razzak

Original articles de Groot, T. et al. Lithium causes G2 arrest of renal principal cells. J. Am. Soc. Nephrol. doi:10.1681/ ASN.2013090988 | Bao, H. et al. Delayed administration of a single dose of lithium promotes recovery from AKI. J. Am. Soc. Nephrol. doi:10.1681/ASN.2013040350 